\title{
The Muonic Hydrogen Lamb Shift Experiment at PSI
}

\author{
F. KOTTMANN ${ }^{1, \star}$, W. AMIR ${ }^{2}$, F. BIRABEN ${ }^{2}$, C. A. N. CONDE ${ }^{3}$, \\ S. DHAWAN ${ }^{7}$, T. W. HÄNSCH ${ }^{5}$, F. J. HARTMANN ${ }^{6}$, V. W. HUGHES ${ }^{7}$, \\ O. HUOT $^{4}$, P. INDELICATO ${ }^{2}$, L. JULIEN ${ }^{2}$, P. KNOWLES ${ }^{4}$, S. KAZAMIAS ${ }^{2}$, \\ Y.-W. LIU ${ }^{7,9}$, F. MULHAUSER ${ }^{4}$, F. NEZ ${ }^{2}$, R. POHL ${ }^{5,9}$, P. RABINOWITZ ${ }^{8}$, \\ J. M. F. DOS SANTOS ${ }^{3}$, L. A. SCHALLER ${ }^{4}$, H. SCHNEUWLY ${ }^{4}$, \\ W. SCHOTT ${ }^{6}$, D. TAQQU ${ }^{9}$ and J. F. C. A. VELOSO ${ }^{3}$ \\ ${ }^{1}$ Labor für Hochenergiephysik, ETH-Hönggerberg, CH-8093 Zürich, Switzerland; \\ e-mail:franz.kottmann@psi.ch \\ ${ }^{2}$ Laboratoire Kastler Brossel, FR-75252 Paris CEDEX 05, France \\ ${ }^{3}$ Departamento de Fisica, Universidade de Coimbra, PT-3000 Coimbra, Portugal \\ ${ }^{4}$ Département de Physique de l'Université Fribourg, CH-1700 Fribourg, Switzerland \\ ${ }^{5}$ Max-Planck-Institut für Quantenoptik, DE-85748 Garching, Germany \\ ${ }^{6}$ Physik-Department, Technische Universität München, DE-85747 Garching, Germany \\ ${ }^{7}$ Physics Department, Yale University, New Haven, CT 06520-8121, USA \\ ${ }^{8}$ Department of Chemistry, Princeton University, Princeton, NJ08544-1009, USA \\ ${ }^{9}$ Paul Scherrer Institute, CH-5232 Villigen, Switzerland
}

\begin{abstract}
A measurement of the $2 S$ Lamb shift in muonic hydrogen $\left(\mu^{-} \mathrm{p}\right)$ is being prepared at the Paul Scherrer Institute (PSI). The goal of the experiment is to measure the energy difference $\Delta E\left(2^{5} P_{3 / 2}-2^{3} S_{1 / 2}\right)$ by laser spectroscopy $(\lambda \approx 6 \mu \mathrm{m})$ to a precision of $30 \mathrm{ppm}$ and to deduce the root mean square (rms) proton charge radius with $10^{-3}$ relative accuracy, 20 times more precise than presently known.

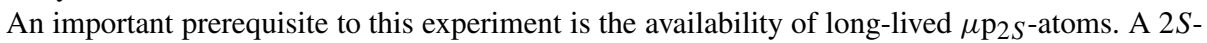
lifetime of $\sim 1 \mu \mathrm{s}$ - sufficiently long to perform the laser experiment - at $\mathrm{H}_{2}$ gas pressures of 1-2 $\mathrm{hPa}$ was deduced from recent measurements of the collisional $2 S$-quenching rate. A new low-energy negative muon beam yields an order of magnitude more muon stops in a small low-density gas volume than a conventional cloud muon beam. A stack of ultra-thin carbon foils is the key element of a fast detector for $\mathrm{keV}$-muons. The development of a $2 \mathrm{keV} \mathrm{X}$-ray detector and a 3-stage laser system providing $0.5 \mathrm{~mJ}$ laser pulses at $6 \mu \mathrm{m}$ is on the way.
\end{abstract}

\section{Introduction}

Since its first discovery in 1947, the Lamb shift in hydrogen and hydrogen-like atoms is the most precisely measured effect of bound-state quantum electrodynamics (QED) [1]. In recent years the determination of the Rydberg constant and Lamb shift became more and more accurate by measuring optical frequencies of

\footnotetext{
^ Corresponding author.
} 
two-photon $1 S \rightarrow 2 S$ and $2 S \rightarrow n S / n D$ transitions [2]. Comparison with theory is now limited mainly by the uncertainty of the proton radius.

The present knowledge of the rms proton charge radius, $r_{p}$, comes mainly from electron scattering experiments [3-6] which are rather difficult to perform and to analyze. The problems of normalization and Coulomb corrections were discussed recently $[7,8]$. An uncertainty of at least $2 \%$ has presently to be attributed to $r_{p}$, corresponding to the relatively wide spread of $r_{p}$-values resulting from different measurements and analysis.

A precise determination of the proton radius is possible by measuring the Lamb shift in muonic hydrogen. The $\mu \mathrm{p}$ Lamb shift, $\Delta E_{2 P-2 S} \approx 0.2 \mathrm{eV}$, is dominated by vacuum polarization which shifts the $2 S$ binding energy towards more negative values. The $\mu \mathrm{p}$ fine- and hyperfine splittings are an order of magnitude smaller than the Lamb shift. The relative contribution of the proton size to $\Delta E_{2 P-2 S}$ is as much as $2 \%$, two orders of magnitude more than for normal hydrogen atoms. $\Delta E_{2 P-2 S}$ was recalculated recently by Pachucki $[9,10]$ considering all QED contributions on the level of a few ppm, including three-loop vacuum polarization $(8 \mu \mathrm{eV})[11]$ and hadronic vacuum polarization $(11 \mu \mathrm{eV})$ [12]. He also found a new value of $12 \pm$ $2 \mu \mathrm{eV}$ for the proton polarization shift, which will ultimately limit the calculated $\Delta E_{2 P-2 S}$-value to the $10 \mathrm{ppm}$ precision level (disregarding terms which depend on the proton radius). Other calculations of this polarization shift resulted in the values $13 \mu \mathrm{eV}$ [13], $17 \pm 4 \mu \mathrm{eV}$ [14], and $16 \mu \mathrm{eV}$ [15]. Measuring the $\mu \mathrm{p}$ Lamb shift opens the field for a future combined analysis of the ep and $\mu$ p experiments, leading to a test of bound-state QED on the ppm level or below, and vacuum polarization up to three-loop contributions.

We are presently setting up an experiment at the Paul Scherrer Institute (PSI) to measure the $\mu \mathrm{p}$ energy difference $\Delta E\left(2^{5} P_{3 / 2}-2^{3} S_{1 / 2}\right)$ in a laser resonance experiment to a precision of $30 \mathrm{ppm}$, which corresponds to $10 \%$ of the natural linewidth. This will allow to deduce the proton radius with $10^{-3}$ relative accuracy, 20 times more precise than presently known. The principle of the experiment is to stop negative muons in $\mathrm{H}_{2}$ gas where highly excited $\mu \mathrm{p}$ atoms are formed. Most of them deexcite quickly to the ground state, but $\sim 1 \%$ form long-lived $\mu \mathrm{p}_{2 S}$ atoms. A short laser pulse with a wavelength tunable around $\lambda \approx 6 \mu \mathrm{m}$ (corresponding to $\left.\Delta E_{2 P-2 S}\right)$ is sent to a mirror cavity surrounding the target gas volume, about $1 \mu \mathrm{s}$ after the muon stop. A resonance curve will be obtained by measuring at different laser wavelengths the number of $2 P-1 S$ transitions (1.9 keV X-rays) which occur in time-coincidence with the laser pulse.

\section{Metastable $2 S$ states of $\mu \mathrm{p}$ atoms}

A number of experiments were performed to investigate the metastability of $\mu \mathrm{p}_{2 S}$ atoms at low gas pressures. From measurements of the $K$-line X-ray yields it can be deduced that about $4 \%$ of the muons reach the $2 S$ state at gas pressures of 
a few $\mathrm{hPa}$ [16-18]. Collisional quenching may deexcite these $\mu \mathrm{p}_{2 S}$ atoms in an interaction with a molecule of the surrounding hydrogen gas:

$$
\mu \mathrm{p}(2 S)+\mathrm{H}_{2} \rightarrow \mu \mathrm{p}(2 P)+\mathrm{H}_{2} \rightarrow \mu \mathrm{p}(1 S)+\mathrm{H}_{2}+K_{\alpha} .
$$

This quenching reaction is energetically allowed only at kinetic energies above $0.31 \mathrm{eV}$, which is the $\mu \mathrm{p}_{2 S} \rightarrow \mu \mathrm{p}_{2 P}$ threshold energy in the laboratory frame. The corresponding calculated cross section is $\sim 10^{-16} \mathrm{~cm}^{2}$ [19] which leads to short $2 S$-lifetimes $(<100 \mathrm{~ns})$ at gas pressures of a few $\mathrm{hPa}$ where a laser resonance experiment is technically feasible. Below threshold the $2 S$-quenching rate is expected to be at least an order of magnitude smaller because any transitions to the $\mu \mathrm{p}$ ground state can occur only during collisions when the $(n=2)$-states are Stark-mixed. The fraction of long-lived $\mu \mathrm{p}_{2 S}$ atoms therefore depends on the distribution of $\mu \mathrm{p}$ kinetic energies which was recently measured at PSI [20]. The conclusion was that $\sim 1 \%$ of the $\mu$ p atoms populate the $2 S$-state at kinetic energies below threshold and are therefore long-lived.

This result was confirmed when a direct signal from long-lived $\mu \mathrm{p}_{2 S}$ atoms was found [21]. A component of $\mu \mathrm{p}_{1 S}$ atoms with kinetic energies as high as $900 \mathrm{eV}$ was measured. It originates from resonant molecule formation from the $2 S$-state and subsequent autodissociation [22]

$$
\mu \mathrm{p}(2 S)+\mathrm{H}_{2} \rightarrow\left\{\left[(\mathrm{pp} \mu)^{+}\right]^{*} \mathrm{pee}\right\}^{*} \rightarrow \mu \mathrm{p}(1 S)+\mathrm{p}+\cdots+1.9 \mathrm{keV} .
$$

From the measured time distributions of $900 \mathrm{eV}-\mu \mathrm{p}_{1 S}$ atoms a $2 S$-quenching rate of $\lambda_{\text {quench }}^{2 S} \approx 5 \times 10^{5} \mathrm{~s}^{-1} \times p_{\mathrm{H}_{2}}[\mathrm{hPa}]$ was deduced [21].

It turns out that the optimum gas pressure for the $2 S-2 P$ laser experiment is $1-2 \mathrm{hPa}$, because the $2 S$-lifetime has to be of the same order as the delay time between muon stop and laser pulse which is nearly $1 \mu \mathrm{s}$.

\section{Beam line for muons at keV-energies}

A new beam line for low-energy negative muons was built at PSI in order to obtain sufficiently high stop rates in a small $\mathrm{H}_{2}$ gas target at pressures of a few $\mathrm{hPa}$. It consists of the cyclotron trap for the production of the low energy muons, the muon extraction channel (MEC) for their transport and selection, and the PSC solenoid with two transmission detectors for the muon trigger (Figure 1).

Negative pions are injected into the cyclotron trap where they decay in flight into muons which are slowed down from $\mathrm{MeV}$ to $\mathrm{keV}$ kinetic energies by a set of thin Formvar foils. An axial electric field ejects the muons along the cyclotron trap axis and out of the trap field region (up to 4 Tesla) [23].

The curved magnetic Muon Extraction Channel (MEC), operated at $0.15 \mathrm{~T}$, transports the muons with typical energies of $20 \mathrm{keV}$ ( $2 \mathrm{MeV} / \mathrm{c}$ momentum) from a region of high neutron background (near the pion beam exit and the cyclotron trap) to the PSC solenoid where the laser resonance experiment will be performed. The MEC acts as a cleaning filter because particles with momenta above $\sim 4 \mathrm{MeV} / \mathrm{c}$ are 


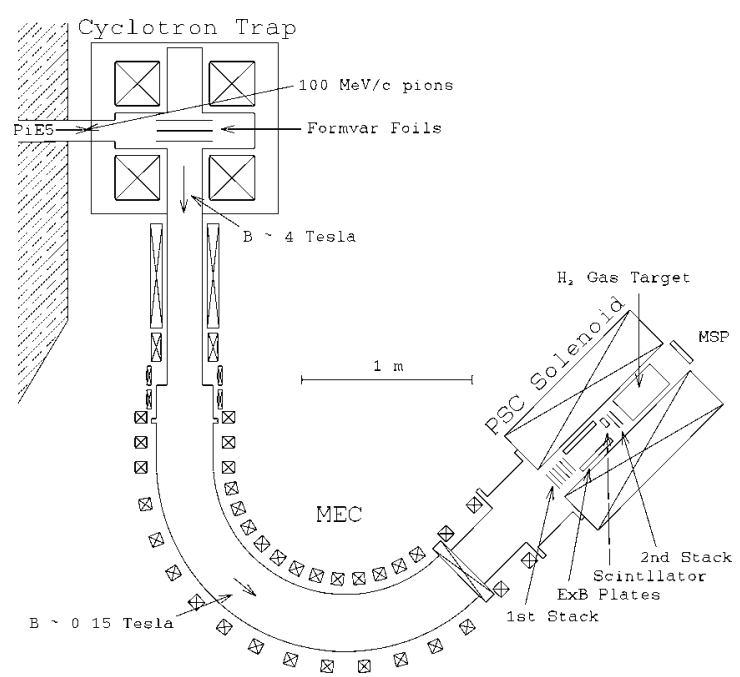

Figure 1. Lay-out of the $\pi \mathrm{E} 5$ area with Cyclotron Trap, magnetic channel MEC, and PSC solenoid. MSP: Microsphere plate.

not transported through the curvature and electrons of keV-energies are separated vertically from the muons by several $\mathrm{cm}$ in the toroidal field. It is therefore possible to remove such electrons which are produced at high rates when charged particles (spiraling in the magnetic bottle field of the trap) cross the extraction foils many times.

The outcoming muons enter the superconducting $5 \mathrm{~T}$ solenoid (PSC) where they first cross a transmission detector suitable for particles with a range of only $\sim 50 \mu \mathrm{g} / \mathrm{cm}^{2}$. It is based on a stack of ultra-thin carbon foils $\left(5 \mu \mathrm{g} / \mathrm{cm}^{2}\right)$ with $1.8 \mathrm{kV}$ voltage between them to compensate for the muon energy loss [24]. Secondary electrons are emitted and accelerated towards a downstream plastic scintillator connected to an external PMT via a long light guide. Between the stack and the scintillator an $\vec{E} \times \vec{B}$ drift space induces a transverse drift (greater for the muons than for the electrons) allowing the muons to pass the scintillator. A muon detection efficiency of $88 \%$ was measured. The foil stack acts simultaneously as a detector for $\mathrm{keV}$-muons and reduces the phase space volume of the beam by frictional cooling [24]. Further downstream the muons cross a second foil stack placed in front of the target. The emitted secondary electrons, accelerated to a few keV, cross the thin gas target and are detected in a downstream microsphere plate (MSP) providing a second muon signal. The stack detectors have time resolutions of better than $10 \mathrm{~ns}$, to be compared with the muon time-of-flight between the two stacks of typically $150 \mathrm{~ns}$. A trigger for the laser will be given by a muon detected in both detectors with the correct time-of-flight.

The rate of muons detected in both stacks and stopped in a $14 \mathrm{~cm}$ long $\mathrm{H}_{2}$ gas target was measured to be $25 \mathrm{~s}^{-1}$ at $2 \mathrm{hPa}$ pressure, for a $7 \times 10 \mathrm{~mm}^{2}$ wide beam. The $\mathrm{e}^{-}$beam contamination is negligible. Optimization of the Formvar extraction 
foils will bring this number to $\sim 100 \mathrm{~s}^{-1}$, about a factor 20 more than what was previously achieved at the best conventional cloud muon beam.

Without the restrictions required by the laser experiment, $1000 \mu^{-} / \mathrm{s}$ with 10 $30 \mathrm{keV}$ kinetic energy and $2 \mathrm{~cm}$ beam diameter were stopped in the Be window of a calibrated Ge diode mounted in the PSC solenoid. $10^{4} \mu^{-} / \mathrm{s}$ are expected for the near future.

\section{The laser resonance experiment}

The $2 \mathrm{keV} \mathrm{X}$-ray detector will consist of two xenon gas proportional scintillation chambers (GPSC) which are insensitive to magnetic field. The $170 \mathrm{~nm}$ secondary scintillation light is detected in a separate $\mathrm{Ar}+\mathrm{CH}_{4}$ gas chamber with CsI-coated microstrip plates [25]. First tests of the chamber in a 5 Tesla field were successful [26]. The use of large area avalanche photo diodes (LAAPD) as detectors for $\mathrm{keV}$ $\mathrm{X}$-rays is also being considered.

The laser system consists of three stages: $\mathrm{A} \mathrm{XeCl} \mathrm{excimer} \mathrm{laser,} \mathrm{a} \mathrm{combination}$ of dye and Ti:Sa laser, and a $\mathrm{H}_{2}$ Raman cell for conversion to $6 \mu \mathrm{m}$ (Figure 2). When a randomly incoming muon is detected by the foil stacks, the laser is triggered. Because of the short $\mu \mathrm{p}_{2 S}$ lifetime the laser light has to arrive in the target within less than $\sim 1 \mu \mathrm{s}$. For this reason our first stage of the laser system consists of a $50 \mathrm{~Hz} \mathrm{XeCl}$ excimer laser delivering $28 \mathrm{~ns}$ long pulses of $600 \mathrm{~mJ}$ at $308 \mathrm{~nm}$. The delay caused by the excimer laser is about $600 \mathrm{~ns}$ from trigger to laser pulse. The second stage, conversion of the $308 \mathrm{~nm}$ light from the excimer laser to the $708 \mathrm{~nm}$ light needed as input of the Raman shifter, is done in two substeps. The $308 \mathrm{~nm}$ light pumps a dye cell, which delivers the $\sim 500 \mathrm{~nm}$ light required to pump the Ti:Sa laser. In the third stage the $708 \mathrm{~nm}$ light is converted to $6.02 \mu \mathrm{m}$ via

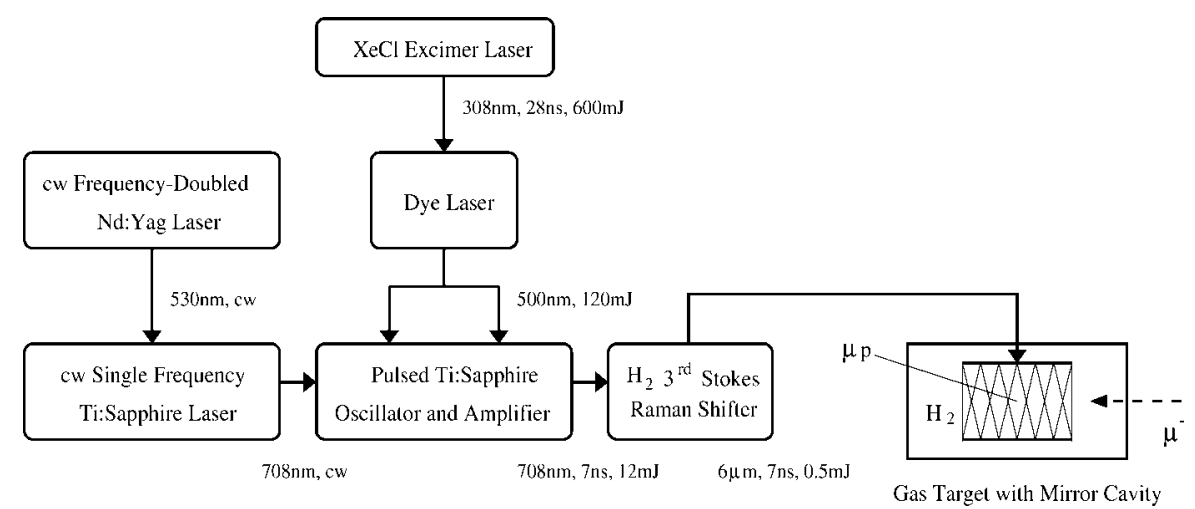

Figure 2. The components of the laser system. The high power $\mathrm{XeCl}$ excimer laser pulse triggered by the muon entrance detector is converted in two steps to a high quality $7 \mathrm{~ns}$ long pulse of $708 \mathrm{~nm}$ which is shifted to the desired $6 \mu \mathrm{m}$ light inside the multipass Raman cell. The light is injected into a mirror cavity to effectively illuminate the muon stop volume inside the PSC solenoid. High resolution frequency selection is provided by injection of a cw Ti:Sa laser. 
third Stokes Raman shift in a high pressure hydrogen cell [27]. The $0.5 \mathrm{~mJ}, 7 \mathrm{~ns}$ long pulses are injected into a multipass cavity inside the gas target in order to effectively illuminate the muon stop region.

First data-taking for the Lamb shift experiment is foreseen for 2002. The expected rate of laser-induced events is a few per hour, on resonance. A precision of $30 \mathrm{ppm}$ for the Lamb shift, resulting in an uncertainty on the proton radius of $10^{-3}$, can be achieved within a reasonably short measuring time.

\section{References}

1. Eides, M. I., Grotch, H. and Shelyuto, V. A., Theory of light hydrogenlike atoms, hep$\mathrm{ph} / 0002158$ (2000).

2. de Beauvoir, B. et al., Eur. Phys. J. D 12 (2000), 61.

3. Hand, L. N., Rev. Mod. Phys. 35 (1963), 335.

4. Simon, G. G. et al., Nucl. Phys. A 333 (1980), 381.

5. Wong, C. W., Int. J. Mod. Phys. 3 (1994), 821.

6. Mergell, P. et al., Nucl. Phys. A 596 (1996), 367.

7. Karshenboim, S. G., Can. J. Phys. 77 (1999), 241.

8. Rosenfelder, R., Phys. Lett. B 479 (2000), 318.

9. Pachucki, K., Phys. Rev. A $\mathbf{5 3}$ (1996), 2092.

10. Pachucki, K., Phys. Rev. A 60 (1999), 3593.

11. Kinoshita, T. and Nio, M., Phys. Rev. Lett. 82 (1999), 3240.

12. Friar, J. L. et al., Phys. Rev. A 59 (1999), 4061.

13. Srartsev, S. A. et al., Phys. Atom. Nucl. 23 (1976), 1233.

14. Rosenfelder, R., Phys. Lett. B 463 (1999), 317.

15. Faustov, R. N. and Martynenko, A. P., AIP Conf. Proc. 564 (2001), 277.

16. Anderhub, H. et al., Phys. Lett. 143B (1984), 65.

17. Egan, P. O. et al., Phys. Rev. A 23 (1981), 1152.

18. Bregant, M. et al., Phys. Lett. A 241 (1998), 344.

19. Jensen, T. and Markushin, V., Report PSI-PR-99-32, nucl-th/0001009 (2000).

20. Pohl, R. et al., Hyp. Interact. 127 (2000), 161.

21. Pohl, R. et al., Hyp. Interact., this issue; Pohl, R., Ph.D. thesis, ETH Zürich No. 14096 (2001), unpublished.

22. Wallenius, J. et al., Hyp. Interact., this issue.

23. de Cecco, P. et al., Nucl. Instrum. Meth. A 394 (1997), 287.

24. Mühlbauer, M. et al., Hyp. Interact. 119 (1999), 305.

25. Veloso, J. F. C. A., dos Santos, J. M. F. and Conde, C. A. N., Nucl. Instrum. Meth. A 422 (1999), 273.

26. Veloso, J. F. C. A. et al., Nucl. Instrum. Meth. A 460 (2001) 297.

27. Rabinowitz, P. et al., IEEE Jour. QE 22 (1986), 797. 